\title{
Framework for acquisition and automated sorting of neural spikes from multielectrode recordings in freely-moving mice
}

Joshua J. Strohl ${ }^{1,2}$, Joseph T. Gallagher ${ }^{1}$, Pedro N. Gómez ${ }^{1}$, Joshua M. Glynn ${ }^{1,2}$, Patricio T. Huerta ${ }^{1,2,3}$

${ }^{1}$ Laboratory of Immune \& Neural Networks, Institute of Molecular Medicine, Feinstein Institutes for Medical Research, Northwell Health, Manhasset, New York, USA, 350 Community Drive, Manhasset, NY 11030, USA.

${ }^{2}$ Department of Molecular Medicine, Zucker School of Medicine at Hofstra/Northwell, 500 Hofstra Blvd, Hempstead, NY 11549, USA.

\section{Email addresses:}

JJ Strohl: jstrohl@northwell.edu

JT Gallagher: jgallagher5@northwell.edu

PN Gomez: pgomez3@northwell.edu

JN Glynn: jglynn@northwell.edu

PT Huerta: pato.huerta@gmail.com

${ }^{3}$ Corresponding author:

Patricio T. Huerta

Feinstein Institute for Medical Research

Laboratory of Immune \& Neural Networks

350 Community Drive

Manhasset, NY 11030

USA 


\section{ABSTRACT}

50 Background: Extracellular recording represents a crucial electrophysiological technique in

51 neuroscience for studying the activity of single neurons and neuronal populations. The electrodes

52 capture voltage traces that, with the help of analytical tools, reveal action potentials ('spikes') as

53 well as local field potentials. The process of spike sorting is used for the extraction of action

54 potentials generated by individual neurons. Until recently, spike sorting was performed with

55 manual techniques, which are laborious and unreliable due to inherent operator bias. As

56 neuroscientists add multiple electrodes to their probes, the high-density devices are able to record

57 hundreds to thousands of neurons simultaneously, making the manual spike sorting process

58 increasingly difficult. The advent of automated spike sorting software has offered a compelling

59 solution to this issue. The purpose of this study is to provide a simple-to-execute framework for

60 using MountainSort, an automated spike sorting pipeline, in conjunction with MATLAB and the

61 acquisition system (Cheetah, Neuralynx). We validate this automated framework with neural

62 recordings from the hippocampus and prelimbic cortex.

63 Methods: Multielectrode recordings of freely-moving mice are obtained from the CA1 region of

64 the hippocampus as they navigate a linear track. Multielectrode recordings are also acquired

65 from the prelimbic cortex, a region of the medial prefrontal cortex, while the mice are tested in a

66 T maze. All animals are implanted with custom-designed, 3D-printed microdrives that carry 16

67 electrodes, which are bundled in a 4-tetrode geometry.

68 Results: We provide an overview of a framework for analyzing single-unit data in which we have

69 concatenated the acquisition system (Cheetah, Neuralynx) with analytical software (MATLAB)

70 and an automated spike sorting pipeline (MountainSort). We give precise instructions on how to

71 implement the different steps of the framework, as well as explanations of our design logic. We

72 validate this framework by comparing manually-sorted spikes against automatically-sorted spikes,

73 using neural recordings of the hippocampus and prelimbic cortex in freely-moving mice. 
74 Conclusions: Automated spike sorting is a necessity for medium and large-scale extracellular 75 neural recordings. Here, we have smoothly integrated MountainSort-based spike sorting into a 76 framework for acquisition and analysis of multielectrode brain recordings in mice.

78 Keywords: Automated spike sorting, spike clustering, MountainSort, Neuralynx, Cheetah, 79 MATLAB, tetrode, electrophysiology, mouse

\section{BACKGROUND}

82 Functional understanding of neural ensembles requires the ability to reliably measure the activity

83 of single neurons, as well as to discriminate the activity of many neighboring neurons, across 84 extended intervals of time (Buzsáki, 2004; Csicsvari, et al., 2003). In vivo electrophysiological 85 techniques with extracellular electrodes that measure action potentials ('spikes') and 86 subthreshold oscillations have been used to record activity from the brain of several mammalian

87 species and have generated a tremendous amount of information (Cacucci et al., 2008; O'Keefe 88 and Dostrovsky, 1971; O'Keefe and Nadel, 1976; Kunz, et al., 2021). Moreover, continuous 89 technological advances have ensured the lasting relevance of this technique (Steinmetz, et al., 90 2021; Wu, et al., 2015). Indeed, brain recordings with multielectrode arrays have been adopted

91 by many neuroscience laboratories all over the world (Yamamoto, et al., 2008). After obtaining a 92 multielectrode recording, spike sorting is a mandatory step for the isolation of neuronal units. This 93 process begins by applying a band-pass filter followed by a voltage threshold to detect all events

94 which fall into the frequency and voltage ranges containing neural spikes. Following event 95 detection, spikes are brought into a spike sorting software package (Rey et al., 2015). Most spike

96 sorting algorithms use dimensionality reduction techniques, such as principal component analysis

97 (PCA), where the high dimensional features of spikes are represented in a 2-dimensional or 3-

98 dimensional space for manual cluster cutting (Gray, et al., 1995; Quirk \& Wilson, 1999). Other 99 software packages implement a template matching approach (Laboy-Juárez, et al., 2019), and 
100 some, such as Spike2 (CED, Cambridge, UK), offer both PCA and template matching options.

101 Events that cluster together are assigned to a unit with an arbitrary label, representing a putative

102 neuron. During this process, clusters or events which are either poorly isolated or likely to 103 correspond to background noise are removed from further analysis.

104 Since the introduction of XCLUST, a pioneering spike clustering program, by Matthew 105 Wilson (Quirk \& Wilson, 1999), several spike sorting packages have been developed using 106 manual or semi-automated approaches. Some commonly used packages include KlustaKwik 107 (Harris, et al., 2000), MClust (Redish et al., 2000), Offline Sorter (Plexon, Dallas, TX), and Spike2 108 (CED). While the manual approach has been the standard for decades, there are notable 109 drawbacks. A significant concern is the lack of reliability, given that different manual operators 110 can have variable outcomes and error rates can be as high as 30\% (Harris, et al., 2000). The 111 operator-dependent nature of the manual approach may thus negatively impact the objectivity 112 and reproducibility of the sorted data (Wood et al., 2004). Another concern with manually-sorted 113 spikes is the amount of time necessary to analyze datasets of any size. Cluster cutting is a time114 intensive process, which effectively acts as a bottleneck in the analysis of acquired neural 115 datasets. Early semi-automated algorithms have continued to rely on human intervention (Harris, 116 et al., 2000; Hill et al., 2012), and some have shown poor accuracy (Pedreira, et al., 2012), 117 resulting in a lasting dependence on manual techniques by the majority of the systems 118 neuroscience community.

119 Recently, automated algorithms have been developed that are both sufficiently accurate 120 and have the ability to sort data obtained from large arrays (Buccino et al., 2020; Chaure et al., 121 2018; Chung et al., 2017; Pachitariu et al., 2016; Rossant et al., 2016). Remarkably, these 122 automated approaches have arisen during a period when advancements in electrode technology 123 have enabled simultaneous recording from hundreds to thousands of densely packed recording 124 sites, from which sorting data would be excruciatingly laborious with previously standard methods 125 (Berényi et al., 2014; Chung, et al., 2019; Steinmetz, et al., 2021). Smaller arrays also benefit 
126 from automated algorithms, as the same approach can be applied to tetrodes for fast and

127 objective results. MountainSort is a particularly attractive package as it has shown to be the most

128 accurate method thus far to sort relatively low-channel-count datasets (Buccino et al., 2020;

129 Chung, et al., 2017; Magland, et al., 2020) and, critically, it requires no user input or changing of

130 parameters across recordings (Chung, et al., 2017). While MountainSort has the potential to be

131 an effective and highly useful spike sorting package, it is still in the development phase and does

132 not have a fully integrated support platform for importing neural recordings obtained across

133 different recording systems and setups. The goal of this study is to provide a simple-to-execute

134 framework for using MountainSort with Neuralynx-acquired neuronal data. Moreover, we validate

135 this framework by comparing manually-sorted spikes against automatically-sorted spikes in

136 neural recordings of the Cornus Ammonis 1 (CA1) region of the hippocampus and the prelimbic

137 (PL) cortex in freely-moving mice.

\section{METHODS}

140 2.1. Ethical statement: Animal experiments were performed in accordance with the National

141 Institutes of Health $(\mathrm{NIH})$ Guidelines under protocols approved by the Feinstein Institutes for

142 Medical Research Institutional Animal Care and Use Committee (IACUC). Our Animal Research

143 Program is registered with the Department of Health and Human Services (DHHS), Office of

144 Laboratory Animal Welfare (OLAW), U.S. Department of Agriculture (USDA \#21R0107), Public

145 Health Service (PHS \#A3168-01) and New York State Department of Health (NYSDOH \#A-060).

146 2.2. Experimental animals: All animals used in this study were male C57BL/6 mice (The

147 Jackson Laboratory, Bar Harbor, ME) of 3 months of age. Mice were maintained on a reverse

148 light cycle (dark: 9:00-21:00) with ad libitum access to food and water. All experiments were

149 carried out during the dark phase of the light cycle. Prior to implanting, mice were housed in

150 groups of four, and were single-housed after implanting. All mice were gently handled prior to

151 surgery (15-min sessions during 3 consecutive days). 
152 2.2. Microdrive preparation: Custom-designed microdrive bodies were fabricated using a 3D

153 printer (Form-2, Formlabs, Somerville, MA). The design of the microdrive body was specific to

154 the brain region being recorded. Polyimide tubing and an electrode-interface-board (Omnetics

155 EIB-16, Neuralynx, Bozeman, MT) were attached to each microdrive body. Tetrodes were wound

156 from $90 \%$ platinum, 10\% iridium wire (diameter $17.8 \mu \mathrm{m}$; California Fine Wire, Grover Beach, CA)

157 and threaded through the polyimide tubes to create a movable 4-tetrode array (Chang, et al.,

158 2013). On the day of implantation surgery, a 'final cut' of the tetrodes was made followed by

159 electroplating with platinum black solution (Neuralynx) to an impedance under $300 \mathrm{k} \Omega$.

160 2.3. Surgery: All surgical procedures were performed under isoflurane anesthesia. The animal's

161 fur was removed from the surgical site, which was then scrubbed with betadine and isopropyl

162 alcohol. An incision was made, exposing the skull, and a layer of C\&B-Metabond (Parkell,

163 Edgewood, NY) was applied and allowed to dry. Two craniotomies were made, one over the

164 cerebellum and the other over the region targeted for tetrode implantation. The coordinates used

165 for dorsal CA1 were $[A P,-2.18, M L,-1.5]$ from bregma, and the coordinates for the PL cortex

166 were $[\mathrm{AP},+1.98, \mathrm{ML},-0.25]$ from bregma. After installing the ground screw into the craniotomy

167 located in the occipital bone, the microdrive was aligned so that the tetrodes were directly above

168 the intended region, and the microdrive was secured in place with dental acrylic. As the dental

169 acrylic hardened, mice were given injection of buprenorphine $(0.05 \mathrm{mg}$ per $\mathrm{kg})$ and saline $(0.5$

$170 \mathrm{~mL}$ ) subcutaneously. Implanted mice were observed for three days following surgery and

171 provided with hydrogel cups containing meloxicam for pain relief. Tetrodes were lowered to their

172 target depth, in CA1 or PL cortex, over the course of the next three days.

173 2.4. Behavioral tasks: Neural recording during behavioral tasks were typically performed 7-10

174 days after the surgery. Mice with tetrodes targeted to CA1 $(n=4)$ were studied in a linear track

175 (80 cm long). For this task, mice experienced a first 'Run' session (moving from one end of the

176 track to the other 16 times; 8 runs 'to the left', 8 runs 'to the right'), a 'Rest' period (10 min in the

177 homecage), and a second 'Run' session (16 times; 8 runs 'to the left', 8 runs 'to the right'), for a 
178 total of 32 runs across the length of the track. Mice with tetrodes targeted to the PL cortex $(n=$ 179 4) were pre-trained in a T maze, before being implanted. The task consisted of running from the 180 start point of the stem toward the decision point and then turning right, or left, to find a sweet food

181 reward that was located at the end of the right arm. Mice were mildly food-deprived (food was 182 removed for 3-4 $\mathrm{h}$ before testing), and were tested in the T maze until they reached a performance 183 accuracy of $75 \%$, which took $3-4$ days (8 trials per day). One week after implantation, animals 184 were tested in the T maze (8 trials in one day).

185 2.5. Data acquisition: Mice were recorded using a headstage pre-amplifier (Neuralynx), which 186 was connected to a programmable amplifier (Lynx-8, Neuralynx) and a Windows PC running the 187 Cheetah acquisition software (Neuralynx). In this study, we used the Cheetah system and a 16188 channel setup comprising of 4 tetrodes, each featuring 4 closely spaced recording channels, 189 along with an overhead camera. The acquisition setup generated 3 datastreams: continuously190 sampled neural signals, discretely-sampled spikes, and the animal's XY location. For the 191 continuous data, the 16 channels were acquired at $30 \mathrm{kHz}$ and contained the voltage for each 192 channel at every timestamp. For the spike data, the 16 channels were first band-pass filtered $193(600 \mathrm{~Hz}$ to $6 \mathrm{kHz})$ and only the fluctuations that surpassed the assigned voltage threshold (120 $194 \mu \mathrm{V}$ ) were captured. For the $\mathrm{XY}$ data, video tracking was achieved via a ceiling-mounted camera 195 which tracked the position of an LED (light-emitting diode) mounted to the headstage in the 196 Cheetah software at $30 \mathrm{~Hz}$. The raw video footage was separately saved as well.

197 2.6. Hardware and software used for data analysis: After acquisition, data were transferred 198 to a Linux machine (running Ubuntu 18.04.5 LTS) for sorting using the automated framework. On 199 the Linux system, data were prepared for sorting with MountainSort, using MATLAB 2017b 200 (MathWorks, Natick, MA), passed through the MountainSort pipeline, and the sorted spikes were 201 saved as text files for further analysis. Spike sorting using the manual method was completed 202 using Spike2 (version 8, Cambridge Electronic Design, Cambridge, UK) on a PC running 203 Windows 10 (Microsoft, Redmond, WA). Sorted spikes were analyzed using NeuroExplorer 
204 version 5 (Plexon, Dallas, TX) and MATLAB on a PC running Windows 10. Final results were 205 processed in MATLAB, Excel 2013 (Microsoft), and Origin 2019 (OriginLab, Northampton, MA).

206 2.7. Statistical analysis: Data are presented as mean \pm standard deviation (SD), or median and 207 quartiles (Q1 and Q3), as indicated. To examine statistical significance, which was defined as $P$ $208<0.05$, we used two-sample ANOVA and Student's t-test in samples that were normally 209 distributed. We also used nonparametric tests, namely Mann-Whitney $U$ test and Kolmogorov210 Smirnov test, in samples that were not normally distributed.

\section{RESULTS}

213 The analysis process for neural data consists of multiple steps, using different software packages

214 as required. A central goal of this work is to provide a roadmap for analysis of single-unit data,

215 with a focus on how we have concatenated the Cheetah system with MATLAB and the automated

216 spike sorting technology, MountainSort. The overall process begins with the acquisition of neural

217 data, followed by spike sorting, and finally, the computation of spike parameters which is done in

218 a manner specific to the phenomenon being studied. The code used in this study can be found

219 at: GitHub: spike sorting.

220 3.1. Description of the Cheetah file formats: The Cheetah system generates three groups of

221 data (Fig. 1). The continuous datastream is saved in the files named CSC1. ncs up to CSC16.ncs

222 (Neuralynx continuously sampled), which comprise the voltage at every timestamp (sample rate,

$22330 \mathrm{kHz}$ ) for each separate channel. The spike datastream is saved in the files named TT1.ntt

224 up to TT4.ntt (Neuralynx tetrode), in which each file represents a tetrode and contains the spike 225 information across the four channels of a particular tetrode. The XY datastream is saved in the 226 file VT1.nvt (Neuralynx video tracking), which contains the position of an LED mounted onto the 227 headstage on the animal's head. 
228 3.2. Overview of the spike sorting methods: Both the automated spike sorting framework and

229 manual spike sorting method follow similar overall trajectories. The raw data are saved by the

230 Cheetah system, then brought into the spike sorting package, in which the spikes are sorted and

231 exported for final analysis in NeuroExplorer and MATLAB, depending on the parameter in 232 question (Fig. 1).

233 For the automated spike sorting framework, only the continuous channels are used (Fig.

234 1a). The ncs files are imported into MATLAB, they are merged, and then converted to the mda

235 format (e.g., raw.mda). The files are then passed through MountainSort where pre-processing 236 and spike sorting occur. Finally, the sorted spikes are saved as text files (e.g., spiketimes.txt)

237 which allows for easy importing into most software packages. We use NeuroExplorer and 238 MATLAB to analyze sorted data. Within NeuroExplorer, the analysis of spikes, along with the 239 position data from the video feed (e.g., VT1.nvt), can be used to generate place field maps, 240 autocorrelograms, and many other visualizations. From that point, the data can be exported into

241 MATLAB for quantitative analysis such as calculation of place field areas or spatial information.

242 For other forms of analysis, sorted spikes and position data are imported directly into MATLAB

243 without passing through NeuroExplorer. Note that in order to import spikes from spiketimes.txt

244 into NeuroExplorer, the correct option for importing data must be set. This can be done as follows:

245 Open NeuroExplorer. Under the View tab, click on Data Import Options. In the window, click on 246 the File Extensions box. Scroll down to txt and select the following option: Text File 247 (pairs<channel $><$ timestamp $>$ ), and then click OK to exit the window.

248 For the manual spike sorting method (Fig. 1b), only the discrete spike channels from 249 Cheetah are imported straight into Spike2 where file merging, spike sorting, and exporting of 250 sorted units are completed. Our team has historically used manual spike sorting platforms such 251 as Offline Sorter or KlustaKwik (Chang and Huerta, 2012; Faust, et al., 2013). In recent years, 252 we have exclusively used Spike2 which has proven to be a versatile software for sorting and 
253 visualizing spike data. In Spike2, we use PCA to generate the components for cluster cutting.

254 The spikes can be easily viewed as either overlaid waveforms or as points across time. If different 255 recording sessions from the same day need to be merged together for sorting and then split back

256 into the original sessions, this is all done within the Spike2 environment. Following completion of 257 manual sorting, the spikes are exported for downstream analysis.

258 3.3. Data preparation for MountainSort: The automated spike sorting process begins with the 25916 continuously-sampled channels which are saved in the ncs format (Fig. 2a). In principle, these 260 files contain the information about local field potential and the spike signals, but the spikes have 261 not been isolated from the rest of the recording yet. The continuous channels are imported into 262 MATLAB and the data files are merged according to the principle that all the recording sessions 263 from a given mouse, obtained in the same day, are joined together. The merged files are then 264 converted from the Neuralynx ncs data format into the mda (multi-dimensional array) format, which 265 is compatible with MountainSort. Merging files is done so that spikes which may originate from 266 the same neurons, can be clustered together, with the use of the m2020_mergeandconvert.m 267 script (Fig. 2). For the sake of simplicity, the script is made to work with data acquired with a 268 configuration of 16 -channels/4 tetrodes, however it can easily be modified to work with other 269 recording configurations. The input for this script follows a simple organization scheme. All of 270 the experiments to be sorted need be placed into a single parent folder. Within the parent folder, 271 recording sessions from the same mouse on the same day are grouped into individual 'mouse \&

272 day' folders (Fig. 2). Recording files go in the session folder with no additional subfolders, as is 273 saved by default by the Cheetah system. Next, the m2020_mergeandconvert. $m$ script is opened

274 in MATLAB. The line asking for the parent folder needs to be changed to reflect the location of 275 the parent folder stated above.

276 The m2020_mergeandconvert. $\mathrm{m}$ script first reorganizes the continuous data on a tetrode277 by-tetrode basis so that each of the 4 recording channels from each tetrode is placed into an 
278 individual subfolder. Next, the ncs files (CSC1.ncs and so on) are imported into the MATLAB

279 environment as variables using the mex files provided by Neuralynx. For each mouse \& day

280 folder, the raw continuous channels are imported and merged into a single [ $1 \mathrm{XM}$ ] variable. These

281 files are then saved into the MountainSort-compatible format, as raw.mda, in which each file

282 contains the 4 channels of a single tetrode for an entire mouse \& day unit of recording. The

283 raw.mda files are placed into folders created for each tetrode (e.g., $t \mathrm{t} 1, \mathrm{tt} 2, \mathrm{tt} 3, \mathrm{tt} 4)$ in the mouse

284 \& day folder (Fig. 2a). Along with the mda files, three other files are generated for each tetrode.

285 The first file, geom.csv, contains the electrode geometry for the recording, which is set to

286 resemble the tetrode recording configuration. The second file, params.json, contains the

287 sampling rate of the recording (in our case, $30 \mathrm{kHz}$ ) as well as the direction of spike occurrences

288 (positive or negative). Both files are necessary for MountainSort. The third file, $t_{-}$info.csv,

289 gives the start and end time of each recording session for the mouse \& day, and it is used (after

290 MountainSort) to separate the merged files back into their original recording sessions (Fig. 2b).

291 After running the m2020_mergeandconvert script, the data are ready for the spike sorting

292 pipeline. Prior to sorting any experiments, MountainSort needs to be installed according to:

293 GitHub: sorting pipeline. After installation, this pipeline can be executed by editing the file titled

294 runsort.sh and inputting each file, on a tetrode-by-tetrode basis. For each line, one needs to

295 add the path of the input folder (as shown in Fig. 3a), followed by the path for the desired output

296 folder, which is generated by the pipeline. One then runs the runsort.sh file that leads

297 MountainSort to input the raw.mda, params.json, and geom.csv files, run the pipeline, and

298 generate a firings.mda output for each tetrode, which contains the timestamps for the firing

299 times for each sorted unit (Fig. 3a).

300 3.4. Interpretation of the sorted spike data: Following MountainSort, the firings.mda files

301 are saved in an appropriate format for further analysis. This is achieved by running the

302 m2020_ms_out_timestamps.m script. Note that this script requires the MATLAB m file 
303 ms_objects.m to be included in the file path as this file contains functions needed to run the

304 script. The merged and sorted files are first split up into sessions as originally recorded (Fig. 3 b).

305 To ensure compatibility with multiple analysis programs, the output is saved as a text file titled

306 spiketimes.txt. This file contains two columns, which make up [unit ID, timestamp] pairs (Fig.

307 3c). This file structure can be imported into a variety of analysis packages including MATLAB

308 and NeuroExplorer. The waveforms are not exported with this dataset, allowing for fast

309 computation and small file sizes. If waveforms need to be viewed, a MATLAB-based waveform

310 viewer is available at: GitHub: matlab waveforms.

311 The waveforms are found by matching the timestamp to the raw.mda data for each

312 isolated unit. From there, the waveforms are saved as mat files in MATLAB and can be plotted

313 as overlaid individual waveforms, or as the average waveform for each single-unit. Other

314 waveform viewing options are currently being developed and may be available on the GitHub

315 page for the Flatiron Institute: GitHub: Flatiron Institute.

316 3.5. Validation of the automated framework with CA1 recordings: Neural signals were

317 recorded in the stratum pyramidale of CA1 in mice $(n=4)$ that were running along a linear track

318 (Fig. 4a). The spiking activity was used to compare the output of the automated framework

319 against a manual sorting method. We show several examples of single-units, chosen randomly,

320 which were obtained with the MountainSort pipeline (Fig. 4b) and manually-sorted with Spike2

321 (Fig. 4c). Manual spike sorting was performed by experienced operators and the final results

322 were checked for quality by two independent observers. Comparison of single-units sorted

323 automatically and manually, during 16 recording sessions (Fig. 4d), revealed that the total number

324 of single-units per session was not significantly different between groups (Fig. 4d, top; automated

$325=32.31 \pm 11.85[$ mean $\pm \mathrm{SD}]$, range $=18-58 ;$ manual $=43.31 \pm 12.35$, range $=23-74 ; t=2.13$,

$326 P=0.05$, paired $t$ test). However, comparison of the spike amplitudes, defined as the peak-to-

327 peak voltage of the averaged waveform from each sorted unit, showed that the automated 
328 framework sorted single-units of significantly higher amplitude than the manual method (Fig. 4d,

329 bottom; automated: median $=0.35, \mathrm{Q} 1-\mathrm{Q} 3=0.22-1.32 ;$ manual: median $=0.19, \mathrm{Q} 1-\mathrm{Q} 3=0.15-$

$330 \quad 0.25 ; d=0.55, Z=3.55, P=3.49 \times 10^{-12}$, Kolmogorov-Smirnov test).

331 We used the place cell properties of the hippocampal single-units sorted automatically

332 (MountainSort pipeline) and manually (using Spike2) for direct comparison of the output from both

333 methods. For illustrative purposes, two pairs of single-units with place fields in close proximity

334 across methods were selected (Fig. 5a, b). In one case (Fig. 5a), the two place cells have similar

335 firing rates as a function of position of the mouse along the linear track (Fig. 5a, left) and display

336 a well-defined place field (at $\sim 70-\mathrm{cm}$ on the track), with the peak firing rate occurring as the mouse

337 moves from right to left. Moreover, their waveforms (Fig. 5a, next-to-left) are highly similar

338 across the 4 channels of the tetrode when comparing the automated and the manual traces. Their

339 autocorrelograms (Fig. 5a, next-to-right) show few refractory period violations but appear

340 different depending on the sorting method, which is likely the result of MountainSort clustering

341 more spikes into the single-unit when compared to manual sorting operators. Analysis of all spike

342 events during a 5-min period shows that the spike amplitudes for the two sets are statistically

343 different (Fig. 5a, right; automated $=0.103 \pm 0.03$ [mean \pm SD]; manual $=0.164 \pm 0.018 ; F=$

$3442.81, P=6.65 \times 10^{-9}$, two-sample ANOVA). Notably, the spikes appear to fire in bursts, as would

345 be expected of hippocampal place cells, with the automated unit showing more pronounced

346 bursting, and also more variance, than the manual unit. This example suggests that the

347 automated framework is more accepting toward clustering spikes of various amplitudes into a

348 single-unit when compared to the manual method. The second example (Fig. 5b) depicts two

349 place cells with multiple place fields, as revealed in their firing profiles (Fig. $\mathbf{5 b}$, left). The primary

350 place field is near the center of the track $(20-40 \mathrm{~cm})$, when the mouse moves from right to left,

351 but relatively high firing rates occur in other regions of the track as well. Although the main place

352 field is similar across sorting methods, the large differences in extraneous activity suggest that

353 the single-units are not optimally clustered. The waveforms (Fig. 5b, next-to-left) show high- 
354 amplitude traces in channels 1 and 2, but the manually-sorted waveforms also have high 355 amplitudes in channels 3 and 4 compared to the automated traces. The autocorrelograms (Fig. 356 5b, next-to-right) show few refractory period violations but the shapes are different, with the 357 automated unit featuring more firing close to zero, which suggests a greater tendency to burst, 358 whereas the manual unit displays a firing pattern that is spread through the autocorrelogram. 359 Analysis of all spike events during a 5-min period of recording shows that the two sets are 360 statistically different (Fig. 5b, right; automated $=0.231 \pm 0.092$ [mean \pm SD]; manual $=0.329 \pm$ $3610.048 ; F=3.575, P=1.65 \times 10^{-46}$, two-sample ANOVA). The bursting nature of the single-units 362 is evident in both methods, but there is more variance in the amplitude of the automated unit. It 363 is possible that in both cases, spikes arising from different neurons were clustered as the same 364 unit. The higher extraneous firing in the manual unit, as well as the differences in the waveforms 365 across sorting methods, strongly suggest that more inappropriate spikes were included in the 366 manual method compared with automated sorting.

367 When counting the number of single-units classified as place cells across 7 sessions in 368 the linear track, we found that the automated and manual sorting methods yielded similar 369 quantities of single-units per recording (Fig. 5c; automated: median =11, Q1-Q3 = 7-15; manual: 370 median = 11, Q1-Q3 = 9-16; $U=21, Z=0.38, P=0.7$, Mann-Whitney $U$ test). Only 2 sessions

371 behaved as outliers, with the number of single-units varying substantially across methods (Fig.

372 5c; automated with 30 vs. manual with 9; automated with 6 vs. manual with 17). While there are

373 similarities and differences among spikes sorted with the two methods, neurons act together in

374 groups, so it is important to view results at the ensemble level as well as that of the individual

375 neuron. By normalizing the firing rates of each unit and sorting all units by the location of their

376 peak firing rate, the activity of place cells in the linear track can be viewed as an ensemble of units

377 (Fig. 5d). We implanted 4 mice and recorded as they ran the length of the track and generated

378 place cell ensembles using each sorting method. Here we find that, at the ensemble level, the

379 activity of the place cells appears similar across both methods. Remarkably, in the 'automated 
spike sorting' ensemble, place fields appear better isolated compared to the 'manual spike sorting'

381 ensemble (Fig. 5d), as suggested by the reduced extraneous activity outside of the main place

382 fields for each unit. Importantly, both sorting paradigms result in ensembles covering the full

383 length of the track.

384 3.6. Validation of the automated framework with PL cortex recordings: We sought to 385 compare the automated framework (MountainSort pipeline) and a manual sorting method 386 (Spike2) using recordings from the PL cortex, a region located within the medial prefrontal cortex.

387 Prior to surgery, the mouse was trained to go to the right arm in the T maze. Tetrodes were 388 implanted into the PL cortex, and the freely-moving mouse was tested in the T maze (Fig. 6a).

389 We examined sorted units based on their activity as the animal navigated from the start point (in 390 the stem) toward the reward (end of the arm). For illustrative purposes, a pair of single-units that 391 seem to have similar properties across methods are shown (Fig. 6b). In this example, the 392 automated unit refers to a neuron that is sorted using MountainSort (Fig. 6b, top) and the manual 393 unit is sorted with the manual method (Fig. 6b, bottom). The waveforms from the automated unit 394 are nearly identical to the waveforms of the manual unit, and the autocorrelograms are similar as 395 well, although the automated unit displays more firing and bursting (Fig. 6b). By plotting the spike 396 activity of the example units as trial-by-trial raster plots, one can observe their dynamic activity 397 (Fig. 6c). Each row of the raster represents the firing of the unit during a trial in the T maze, and 398 the center of the plot corresponds to the time when the mouse is at the intersection of the stem 399 and the arms of the T maze (yellow line in Fig. 6a, c). Statistical comparison shows that the 400 numbers of isolated spikes per trial are not significantly different between the two sets (Fig. 6c; 401 automated: median $=27.5, \mathrm{Q} 1-\mathrm{Q} 3=14.25-42.5 ;$ manual: median $=15.5, \mathrm{Q} 1-\mathrm{Q} 3=13.25-23.5$; $402 U=45, Z=1.315, P=0.189$, Mann-Whitney $U$ test). Notably, the automated unit shows quite a 403 similar pattern of activity across multiple trials, which highlights the reliability of the automated 404 method in isolating a well-behaved unit. In contrast, the manual unit displays a more widespread 405 pattern of spiking across the trials, which would be indicative of a poorly-isolated neuron. 


\section{DISCUSSION}

408 We have designed a novel framework to smoothly combine the acquisition of neuronal signals

409 from multielectrode recordings of freely-moving mice, using the Cheetah system (Neuralynx), to 410 the automated spike sorting pipeline MountainSort (Chung et al., 2017). It is clear that automated 411 spike sorting has become a necessity for medium and large-scale extracellular neural recordings, 412 as it involves the extraction of (ideally) all the action potentials generated by an individual neuron 413 from an ocean of activity in the extracellular recordings. We provide a detailed roadmap of the 414 steps, from data acquisition to file managing between the different software platforms (Cheetah, 415 MATLAB, MountainSort, and NeuroExplorer). In short, our solution provides full integration of 416 MountainSort-based spike sorting to Cheetah-based acquisition of neuronal signals in mice.

417 As this is intended to be a complete framework compatible with existing analysis software, 418 we then convert sorted spikes from the mda format used by MountainSort to a simple txt format. 419 While there is indeed a network of analysis tools being developed around MountainSort, these 420 packages are still in the development phase. Furthermore, many established labs need a way to 421 seamlessly implement state-of-the-art automated spike sorting methods in a manner compatible 422 with existing analysis processes. In our case, we use NeuroExplorer and MATLAB, both of which 423 are widely used programs for the analysis of neural data. Thus, we have created an original end424 to-end framework to integrate the automated spike sorting technology of MountainSort with 425 Cheetah-acquired recordings into a data analysis framework consisting of widely-used software 426 packages.

427 There are several benefits to using the automated pipeline. Critically, automated spike 428 sorting provides a repeatable and objective methodology, in contrast to manual methods which 429 might be quite subjective. Manually sorting spikes yields variable datasets among different 430 operators, even when the operators have significant experience with the technique. Another 431 important benefit regards to the amount of time taken to sort datasets of almost any size. 
432 Manually sorting is a slow and user-intensive process. Each recording can take a period of user-

433 input time ranging from several minutes up to a few hours to finish, making for a time-intensive

434 process. In contrast, using the automated framework, multiple recordings can be quickly sorted

435 in a single run. In this scenario, MountainSort processes each recording in a period of time shorter

436 than the recording itself, but the user does not need to be actively working during this time period.

437 The user only needs to set up the files to be recorded, reducing the user-input time to mere 438 minutes per recording.

439 We have compared the results of the automated spike sorting framework with a manual 440 spike sorting platform we have used for several years in our laboratory. Using either approach, 441 we can isolate single-unit activity in two different brain regions, the hippocampus and the PL 442 cortex. When we compared the number of units sorted per session, we found similar numbers of 443 automated units and manual units for most sessions, but there were some exceptions. In 444 particular, there were two sessions that stood out as outliers. For one of these outliers, the 445 automated framework sorted more single-units, whereas for the other, the manual method sorted 446 more single-units. While there may be differences at the level of each single-unit, we can identify 447 units isolated with similar waveforms across both algorithms. These units also show similar 448 activity in behaviorally relevant tasks such as the linear track or T maze. Furthermore, when 449 viewed at the ensemble level, the datasets look largely similar with only slight variations in the 450 precision of firing.

451 We think that automated spike sorting platforms are poised to become a critical 452 component in the neuroscientist's toolkit, considering that within the next decade, we will very 453 likely experience an exponential increase in the size of the recording arrays (Alivisatos et al. 2013;

454 Steinmetz et al., 2021). With the next generation of brain probes including up to thousands of 455 recording sites, the sheer complexity of the datasets is bound to be too large for manual sorting, 456 or even semi-automated methods, to be capable of processing them. It seems apparent that 457 automated spike sorting needs to become easy to use and properly validated across different 
458 acquisition setups, brain regions and mammalian species. In this respect, we have presented a

459 complete automated framework for sorting neural spike data acquired from Cheetah-based 460 systems.

\section{5. CONCLUSIONS}

463 - We present a complete framework for automated spike sorting using the MountainSort 464 package, with all the code freely-available in the GitHub repository.

465 - The toolset delivers automatically-sorted spikes from recordings obtained with a Cheetah466 based system, and exports the sorted spikes in a format which is highly compatible with $467 \quad$ existing data analysis routines.

468 - Integration of automated spike sorting software is a critical step in the analysis of neural data, 469 and becomes increasingly important as the size and complexity of datasets continues to $470 \quad$ increase with new electrode technology.

471 - An improved understanding of the neural signals obtained with multielectrode recordings 472 within the brain will be paramount for any bioelectronic approaches targeting brain systems.

\section{$474 \quad$ List of abbreviations}

475 AP, anterior / posterior

476 CA1 - Cornus Ammonis 1

$477 \mathrm{~cm}$, centimeter

$478 \mathrm{~Hz}$, hertz

$479 \mathrm{~kg}$, kilogram

$480 \mathrm{kHz}$, kilohertz

$481 \mathrm{k} \Omega$, kilo-ohms

482 LED, light-emitting diode 
483 mg, milligram

$484 \mathrm{ML}$, medial / lateral

485 min, minute

$486 \mathrm{mV}$, millivolt

487 PL, prelimbic cortex

488 s, second

DECLARATIONS

493 Ethics approval: All animal experimentation was performed in accordance with the National 494 Institutes of Health (NIH) Guidelines, under protocols approved by the Institutional Animal Care 495 and Use Committee (IACUC) of the Feinstein Institute for Medical Research. Our Animal 496 Research Program is registered with the Department of Health \& Human Services (DHHS), Office 497 of Laboratory Animal Welfare (OLAW), United States Department of Agriculture (USDA 498 \#21R0107), Public Health Service (PHS \#A3168-01) and New York State Department of Health 499 (NYSDOH \#A-060).

500

501 Consent for publication: Not applicable

503 Availability of data and material: The datasets used and analyzed during the current study are 504 available from the corresponding author on reasonable request. Code used in this manuscript 505 may be found at the following addresses:

506 https://github.com/HuertaLab/spike sorting. DOI: 10.5281/zenodo.5213877

507 https://github.com/HuertaLab/spike sorting/tree/main/sorting pipeline

508 https://github.com/HuertaLab/spike sorting/tree/main/matlab waveforms 
510 Competing interests: The authors declare that they have no competing interests

512 Funding: This work was supported by the National Institute of Health (NIH) grant 5P01Al102852

513 and NIH grant 5P01AI073693, as well as DOD Grant, to PTH.

515 Authors' contributions: JJS, JTG, PNG, JMG, PTH contributed equally to the completion of this 516 work, including conceptualization, data acquisition and data analysis. JJS wrote the software 517 code and MATLAB scripts. PTH assembled the final figures. JJS and PTH prepared the 518 manuscript. The authors read and approved the final manuscript, and agreed to its publication.

520 Acknowledgements: We are grateful to Elvira Strohl for the schematic drawings (figures 4 and 521 6). We thank Jeremy Magland for helpful discussions about the MountainSort software platform.

\section{REFERENCES}

526 Alivisatos AP, Andrews AM, Boyden ES, Chun M, Church GM, Deisseroth K, Donoghue JP, 527 Fraser SE, Lippincott-Schwartz J, Looger LL, Masmanidis S, McEuen PL, Nurmikko AV, Park H, 528 Peterka DS, Reid C, Roukes ML, Scherer A, Schnitzer M, Sejnowski TJ, Shepard KL, Tsao D, 529 Turrigiano G, Weiss PS, Xu C, Yuste R, Zhuang X. Nanotools for neuroscience and brain activity 530 mapping. ACS Nano. 2013; 7: 1850-1866. doi:10.1021/nn4012847. PMID: 23514423 PMCID: 531 PMC3665747.

533 Berényi A, Somogyvári Z, Nagy AJ, Roux L, Long JD, Fujisawa S, Stark E, Leonardo A, Harris 534 TD, Buzsáki G. Large-scale, high-density (up to 512 channels) recording of local circuits in 
535 behaving animals. J Neurophysiol. 2014 Mar;111(5):1132-49. doi: 10.1152/jn.00785.2013. PMID:

536 24353300; PMCID: PMC3949233.

538 Buccino AP, Hurwitz CL, Garcia S, Magland J, Siegle JH, Hurwitz R, Hennig MH. Spikelnterface, 539 a unified framework for spike sorting. Elife. 2020 Nov 10;9:e61834. doi: 10.7554/eLife.61834.

540 PMID: 33170122; PMCID: PMC7704107.

541

542 Buzsáki G. Large-scale recording of neuronal ensembles. Nat Neurosci. 2004 May;7(5):446-51.

543 doi: 10.1038/nn1233. PMID: 15114356.

544

545 Cacucci F, Yi M, Wills TJ, Chapman P, O'Keefe J. Place cell firing correlates with memory deficits 546 and amyloid plaque burden in Tg2576 Alzheimer mouse model. Proc Natl Acad Sci U S A. 2008

547 Jun 3;105(22):7863-8. doi: 10.1073/pnas.0802908105. PMID: 18505838; PMCID: PMC2396558.

549 Chang EH, Huerta PT. Neurophysiological correlates of object recognition in the dorsal 550 subiculum. Front Behav Neurosci. 2012 Jul 19;6:46. doi: 10.3389/fnbeh.2012.00046. PMID: $55122833721 ;$ PMCID: PMC3400129.

553 Chang EH, Frattini SA, Robbiati S, Huerta PT. Construction of microdrive arrays for chronic neural 554 recordings in awake behaving mice. J Vis Exp. 2013 Jul 5;(77):e50470. doi: 10.3791/50470. 555 PMID: 23851569; PMCID: PMC3731431.

556

557 Chaure FJ, Rey HG, Quian Quiroga R. A novel and fully automatic spike-sorting implementation 558 with variable number of features J Neurophysiol. 2018 Oct 1;120(4):1859-1871. doi: 559 10.1152/jn.00339.2018. PMID: 29995603 PMCID: PMC6230803. 
561 Chung JE, Joo HR, Fan JL, Liu DF, Barnett AH, Chen S, Geaghan-Breiner C, Karlsson MP,

562 Karlsson M, Lee KY, Liang H, Magland JF, Pebbles JA, Tooker AC, Greengard LF, Tolosa VM,

563 Frank LM. High-Density, Long-Lasting, and Multi-region Electrophysiological Recordings Using

564 Polymer Electrode Arrays. Neuron. 2019 Jan 2;101(1):21-31.e5. doi:

565 10.1016/j.neuron.2018.11.002. PMID: 30502044; PMCID: PMC6326834.

566

567 Chung JE, Magland JF, Barnett AH, Tolosa VM, Tooker AC, Lee KY, Shah KG, Felix SH, Frank

568 LM, Greengard LF. A Fully Automated Approach to Spike Sorting. Neuron. 2017 Sep

569 13;95(6):1381-1394.e6. doi: 10.1016/j.neuron.2017.08.030. PMID: 28910621; PMCID:

570 PMC5743236.

571

572 Csicsvari J, Jamieson B, Wise KD, Buzsáki G. Mechanisms of gamma oscillations in the

573 hippocampus of the behaving rat. Neuron. 2003 Jan 23;37(2):311-22. doi: 10.1016/s0896-

574 6273(02)01169-8. PMID: 12546825.

575

576 Faust TW, Robbiati S, Huerta TS, Huerta PT. Dynamic NMDAR-mediated properties of place

577 cells during the object place memory task. Front Behav Neurosci. 2013 Dec 17;7:202. doi:

578 10.3389/fnbeh.2013.00202. PMID: 24381547; PMCID: PMC3865705.

579

580 Gray CM, Maldonado PE, Wilson M, McNaughton B. Tetrodes markedly improve the reliability 581 and yield of multiple single-unit isolation from multi-unit recordings in cat striate cortex. J Neurosci

582 Methods. 1995 Dec;63(1-2):43-54. doi: 10.1016/0165-0270(95)00085-2. PMID: 8788047.

583

584 GitHub: Flatiron Institute.

585 https://github.com/flatironinstitute. Accessed 17 August 2021. 
587 GitHub: matlab_waveforms.

588 https://github.com/HuertaLab/spike sorting/tree/main/matlab waveforms. Accessed 17 589 August 2021.

590

591 GitHub: sorting_pipeline.

592 https://github.com/HuertaLab/spike sorting/tree/main/sorting pipeline. Accessed 17 593 August 2021.

594

595 GitHub: spike_sorting.

596 https://github.com/HuertaLab/spike sorting. Accessed 17 August 2021.

597

598 Harris KD, Henze DA, Csicsvari J, Hirase H, Buzsáki G. Accuracy of tetrode spike separation as 599 determined by simultaneous intracellular and extracellular measurements. J Neurophysiol. 2000 600 Jul;84(1):401-14. doi: 10.1152/jn.2000.84.1.401. PMID: 10899214.

601

602 Hill DN, Mehta SB, Kleinfeld D. UltraMegaSort 2000 Manual. 2012.

603

604 Kunz L, Brandt A, Reinacher PC, Staresina BP, Reifenstein ET, Weidemann CT, Herweg NA, 605 Patel A, Tsitsiklis M, Kempter R, Kahana MJ, Schulze-Bonhage A, Jacobs J. A neural code for 606 egocentric spatial maps in the human medial temporal lobe. Neuron. 2021 Jul 7:S0896607 6273(21)00460-8. doi: 10.1016/j.neuron.2021.06.019. Epub ahead of print. PMID: 34265253.

608

609 Laboy-Juárez KJ, Ahn S, Feldman DE. A normalized template matching method for improving 610 spike detection in extracellular voltage recordings. Sci Rep. 2019 Aug 19;9(1):12087. doi:

611 10.1038/s41598-019-48456-y. Erratum in: Sci Rep. 2019 Nov 19;9(1):17413. PMID: 31427615;

612 PMCID: PMC6700190. 
614 Magland J, Jun JJ, Lovero E, Morley AJ, Hurwitz CL, Buccino AP, Garcia S, Barnett AH.

615 SpikeForest, reproducible web-facing ground-truth validation of automated neural spike sorters.

616 Elife. 2020 May 19;9:e55167. doi: 10.7554/eLife.55167. PMID: 32427564; PMCID: PMC7237210.

618 O'Keefe J, Dostrovsky J. The hippocampus as a spatial map. Preliminary evidence from unit 619 activity in the freely-moving rat. Brain Res. 1971 Nov;34(1):171-5. doi: 10.1016/0006620 8993(71)90358-1. PMID: 5124915.

622 O’Keefe J, Nadel L. The hippocampus as a cognitive map. Oxford University Press. 1976.

624 Pachitariu M, Steinmetz N, Kadir S, Carandini M, Harris K. Kilosort: realtime spike-sorting for 625 extracellular electrophysiology with hundreds of channels. bioRxiv. 2016. 626 http://dx.doi.org/10.1101/061481.

628 Pedreira C, Martinez J, Ison MJ, Quian Quiroga R. How many neurons can we see with current 629 spike sorting algorithms? J Neurosci Methods. 2012 Oct 15;211(1):58-65. doi: 630 10.1016/j.jneumeth.2012.07.010. Epub 2012 Jul 25. PMID: 22841630; PMCID: PMC3657693.

632 Quirk MC, Wilson MA. Interaction between spike waveform classification and temporal sequence 633 detection. J Neurosci Methods. 1999 Dec 15;94(1):41-52. doi: 10.1016/s0165-0270(99)00124-7. 634 PMID: 10638814.

636 Redish AD, Rosenzweig ES, Bohanick JD, McNaughton BL, Barnes CA. Dynamics of 637 hippocampal ensemble activity realignment: time versus space. J Neurosci. 2000 Dec 
15;20(24):9298-309. doi: 10.1523/JNEUROSCI.20-24-09298.2000. PMID: 11125009 PMCID:

639 PMC6772998.

640

641 Rey HG, Pedreira C, Quian Quiroga R. Past, present and future of spike sorting techniques. Brain

642 Res Bull. 2015 Oct;119(Pt B):106-17. doi: 10.1016/j.brainresbull.2015.04.007. PMID: 25931392;

643 PMCID: PMC4674014.

644

645 Rossant C, Kadir SN, Goodman DFM, Schulman J, Hunter MLD, Saleem AB, Grosmark A, 646 Belluscio M, Denfield GH, Ecker AS, Tolias AS, Solomon S, Buzsáki G, Carandini M, Harris KD.

647 Spike sorting for large, dense electrode arrays. Nat Neurosci. 2016 Apr;19(4):634-641. doi:

648 10.1038/nn.4268. PMID: 26974951; PMCID: PMC4817237.

649

650 Steinmetz NA, Aydin C, Lebedeva A, Okun M, Pachitariu M, Bauza M, Beau M, Bhagat J, Böhm 651 C, Broux M, Chen S, Colonell J, Gardner RJ, Karsh B, Kloosterman F, Kostadinov D, Mora-Lopez 652 C, O'Callaghan J, Park J, Putzeys J, Sauerbrei B, van Daal RJJ, Vollan AZ, Wang S, 653 Welkenhuysen M, Ye Z, Dudman JT, Dutta B, Hantman AW, Harris KD, Lee AK, Moser El, 654 O'Keefe J, Renart A, Svoboda K, Häusser M, Haesler S, Carandini M, Harris TD. Neuropixels 2.0: 655 A miniaturized high-density probe for stable, long-term brain recordings. Science. 2021 Apr 656 16;372(6539):eabf4588. doi: 10.1126/science.abf4588. PMID: 33859006; PMCID: PMC8244810.

658 Wood F, Black MJ, Vargas-Irwin C, Fellows M, Donoghue JP. On the variability of manual spike 659 sorting. IEEE Trans Biomed Eng. 2004 Jun;51(6):912-8. doi: 10.1109/TBME.2004.826677. PMID: 66015188858.

661 
662 Yamamoto J, Wilson MA. Large-scale chronically implantable precision motorized microdrive 663 array for freely behaving animals. J Neurophysiol. 2008 Oct;100(4):2430-40. doi: 664 10.1152/jn.90687.2008. PMID: 18667539; PMCID: PMC2576215.

FIGURE LEGENDS

Figure 1. Overview of data analysis procedure. a, Automated spike sorting using MountainSort.

670 Continuously sampled signals are collected in Cheetah, prepared for the MountainSort pipeline 671 in MATLAB, sorted in MountainSort, and finally passed through MATLAB again prior to final 672 analysis. b, Manual spike sorting using Spike2. Discrete spike channels are imported into 673 Spike2, where all preprocessing takes place. Exported spikes are used for final analysis.

675 Figure 2. Merging and converting data prior to automated spike sorting. a, Organization and 676 preparation of data for MountainSort pipeline. Sessions from the same mouse on the same day 677 are placed into the same folder, with the data files organized in each session folder just as they 678 are saved by Cheetah. The parent folder containing all of the 'mouse \& day' subfolders is input 679 into MATLAB to merge and convert the data. The output files are organized by tetrode, and saved 680 in the mouse \& day folder as they contain the signals acquired for all sessions within the folder. 681 b, The m2020_mergeandconvert.m script imports the CSC.ncs files and saves the merged data 682 as raw.mda. Three other files are saved; params.json contains the sample rate and spike 683 direction, geom.csv indicates the tetrode geometry, and t_info.csv contains the start and end 684 times of each recording that are used to split the merged files for session-by-session analysis. 685 The lower panel depicts the m2020_mergeandconvert.m script to be run. 
687 Figure 3. Automated sorting framework. a, The files raw.mda, params.json, and geom.csv are 688 imported into the MountainSort pipeline, which includes both pre-processing and spike sorting.

689 The MountainSort pipeline outputs firings.mda files, which contain the unit IDs and timestamps

690 of sorted spikes. The designated input and output folders are entered into the runsort . sh script,

691 as depicted in the bottom of the panel. b, Using the m2020_ms_out_timestamps.m script, sorted

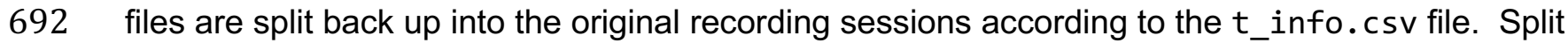
693 files are saved as spiketimes.txt, for easy transfer to other software package for final analysis.

694 The m2020_ms_out_timestamps.m script is shown in the bottom of the panel. c, Output 695 spiketimes.txt files are formatted as two columns of unit ID, timestamp pairs.

697 Figure 4. Validation of the automated framework with hippocampal recordings. a, Schematic 698 depicting tetrode placement in the CA1 region (indicated by yellow oval) and recording paradigm 699 in the linear track. b, Representative waveform overlays of single-units sorted using the 700 automated framework. c, Waveform overlays of single-units sorted using the manual method. d,

701 Top, number of single-units sorted per recording session using the automated framework (AF, 702 green) or manual spike sorting (MSS, black). Bottom, amplitudes of mean waveforms sorted 703 using the automated framework (green) or manual method (black).

705 Figure 5. Hippocampal CA1 recordings in freely-moving mice. a, b, Representative single-units 706 showing the firing rates along the length of the track (left panels), average waveform in each 707 channel of a tetrode (next-to-left panels), autocorrelogram (next-to-right panels; pink lines 708 represent refractory period), and amplitudes of spikes over time (right panels). Automated units 709 are displayed in green and manual units are shown in black. a, Representative single-units likely 710 representing the same putative neuron sorted with automated and manual methods. b, Similar 711 units likely to represent different neurons across automated and manual methods. c, Number of 
712 single-units per recording session using the automated framework (green) or the manual method

713 (black). d, Population of place cells recorded from mice $(n=4)$ running the linear track. Each

714 row represents normalized spike activity of one single-unit, where rows are organized by the

715 position of the peak firing rate for each unit along the linear track. Sorted populations of place

716 cells appear similar across automated and manual methods.

718 Figure 6. Prelimbic cortex recordings in freely-moving mice. a, Schematic depicting tetrode

719 placement in the PL cortex and recording paradigm in the T maze. The yellow line indicates the

720 point at which the mouse chooses to go left or right. b, Representative average waveforms and

721 autocorrelograms of sorted units, likely representing the same putative neuron, across automated

722 and manual methods; TT1_1, and so on, indicate tetrode channel numbers. The pink line

723 represents the refractory period. c, Raster plots for single-units in panel b. Each row represents

724 a trial in the T maze and each tick is a spike. Notice that the trials are aligned to the timepoint

725 indicated by the yellow line (4 s), which corresponds to the moment that the mouse arrives at the

726 intersection of the stem with the arms of the T maze in each trial (as in panel a).

727

728

729

730

731

732

733

734

735

736 


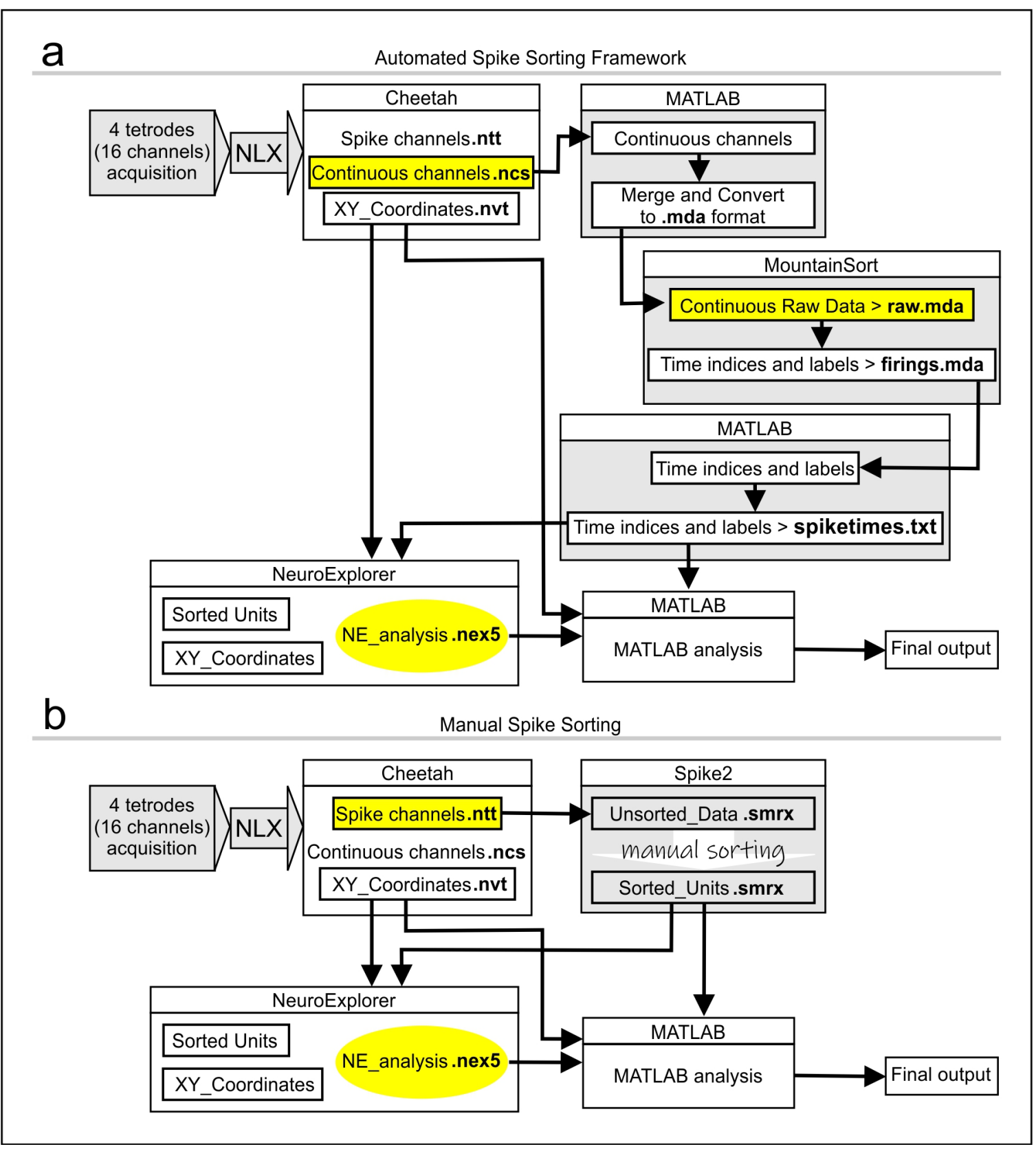

Figure 1. Overview of data analysis procedure. a, Automated spike sorting using MountainSort. Continuously sampled signals are collected in Cheetah, prepared for the MountainSort pipeline in MATLAB, sorted in MountainSort, and finally passed through MATLAB again prior to final analysis. b, Manual spike sorting using Spike2. Discrete spike channels are imported into Spike2, where all preprocessing takes place. Exported spikes are used for final analysis. 


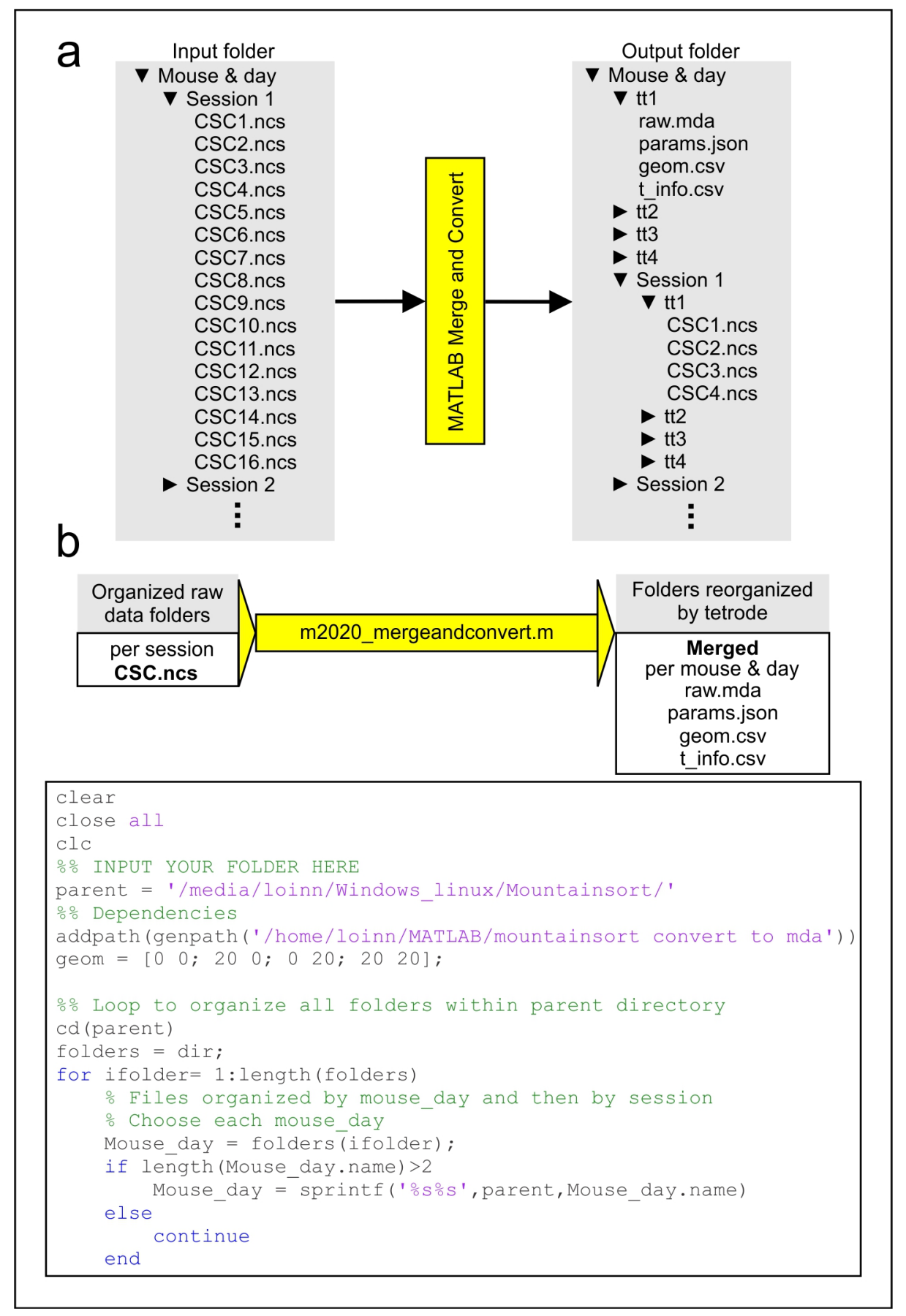

Figure 2. Merging and converting data prior to automated spike sorting. a, Organization and preparation of data for MountainSort pipeline. Sessions from the same mouse on the same day are placed into the same folder, with the data files organized in each session folder just as they are saved by Cheetah. The parent folder containing all of the 'mouse \& day' subfolders is input into MATLAB to merge and convert the data. The output files are organized by tetrode, and saved in the mouse $\&$ day folder as they contain the signals acquired for all sessions within the folder. b, The m2020_mergeandconvert.m script imports the CSC.ncs files and saves the merged data as raw.mda. Three other files are saved; params.json contains the sample rate and spike direction, geom.csv indicates the tetrode geometry, and t info.csv contains the start and end times of each recording that are used to split the merged files for session-by-session analysis. The lower panel depicts the m2020_mergeandconvert.m script to be run. 


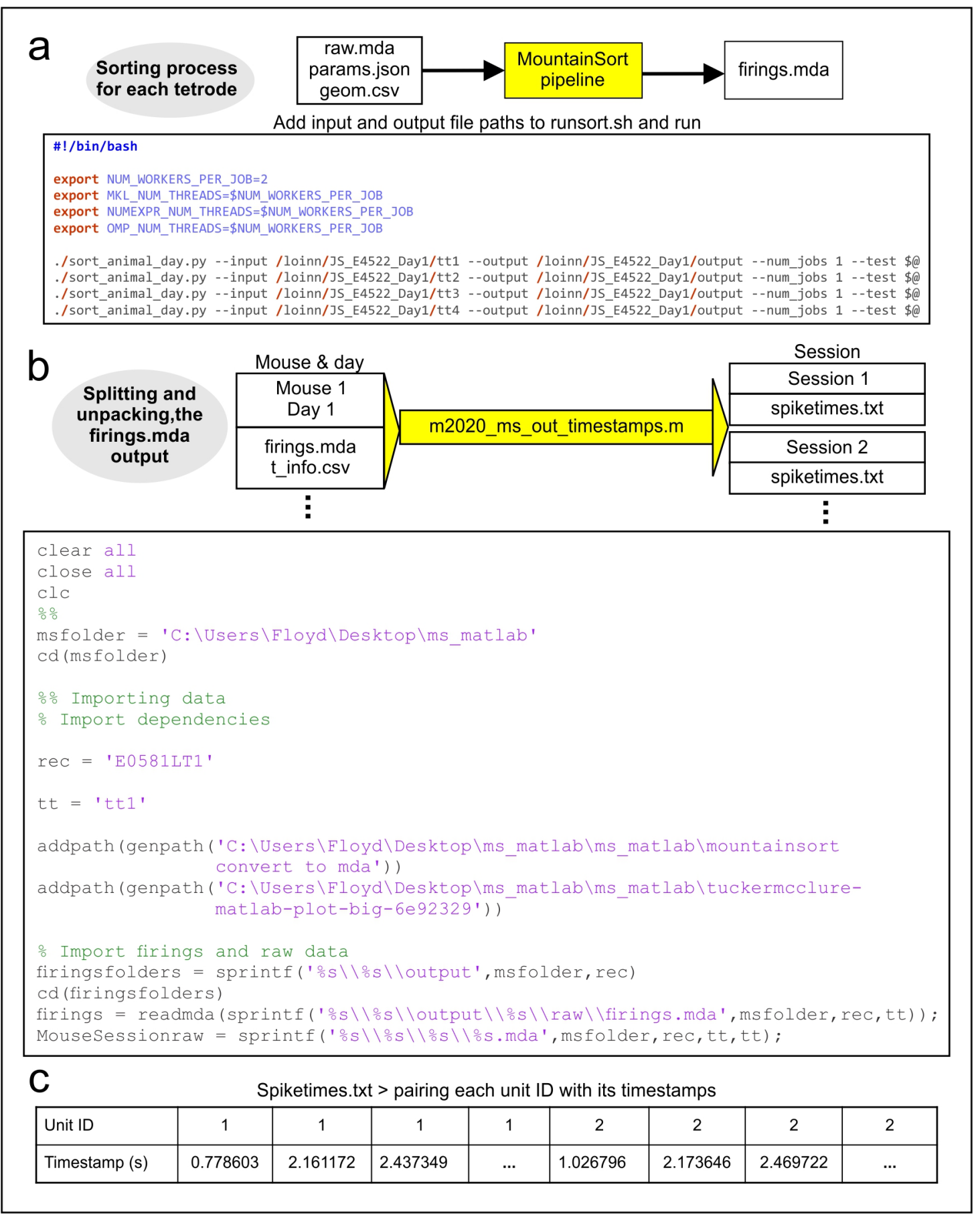

Figure 3. Automated sorting framework. a, The files raw.mda, params.json, and geom.csv are imported into the MountainSort pipeline, which includes both pre-processing and spike sorting. The MountainSort pipeline outputs firings.mda files, which contain the unit IDs and timestamps of sorted spikes. The designated input and output folders are entered into the runsort.sh script, as depicted in the bottom of the panel. b, Using the m2020_ms_out_timestamps.m script, sorted files are split back up into the original recording sessions according to the $t$ _info.csv file. Split files are saved as spiketimes.txt, for easy transfer to other software package for final analysis. The m2020_ms_out_timestamps.m script is shown in the bottom of the panel. c, Output spiketimes.txt files are formatted as two columns of unit ID, timestamp pairs. 
a
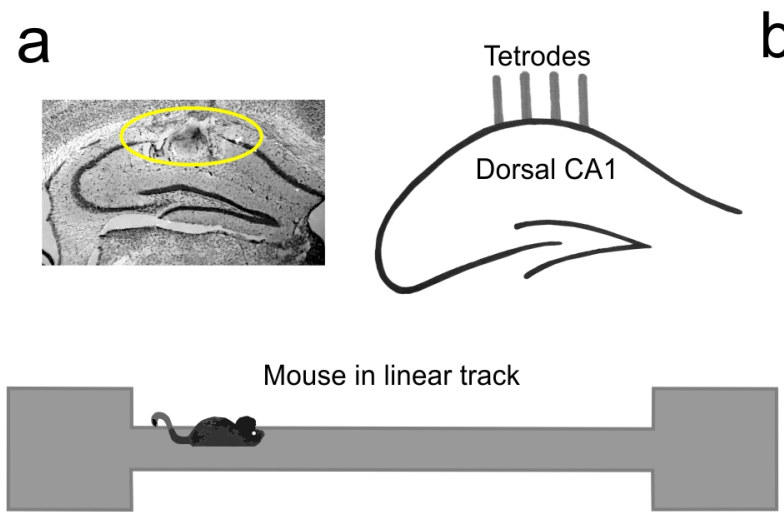

d
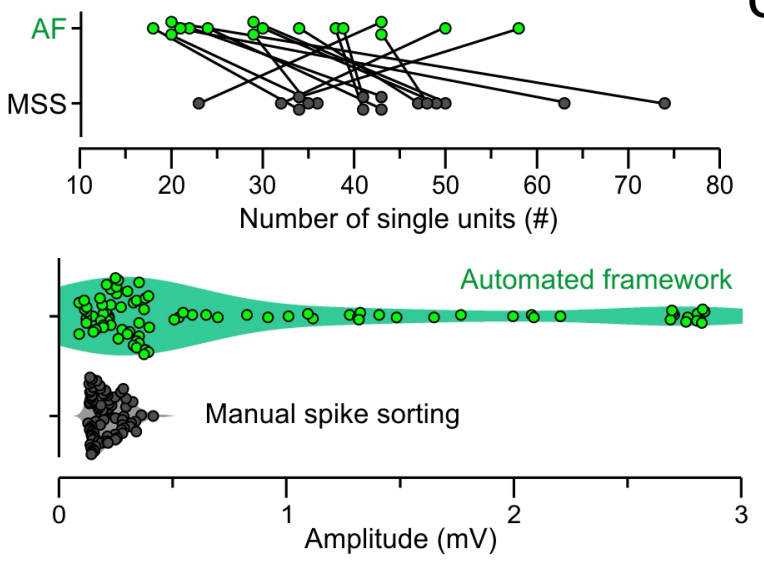

b

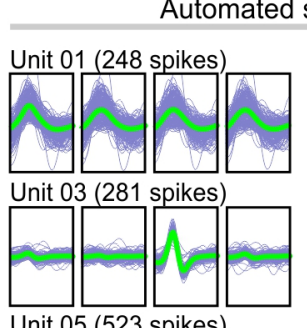

Unit 05 (523 spikes)

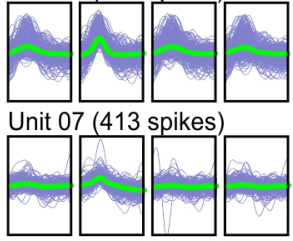

Manual spike sorting

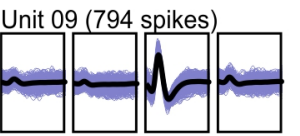

Unit 11(467 spikes)

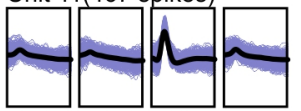

Unit 13 (641 spikes)

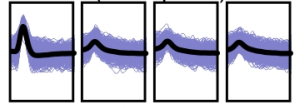

Unit 15 (481 spikes

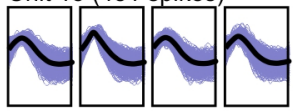

Unit 02 (523 spikes)

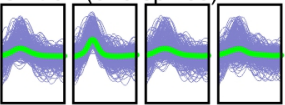

Unit 04 (189 spikes)



Unit 06 (330 spikes)

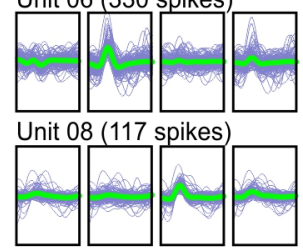

Unit 08 (117 spikes)

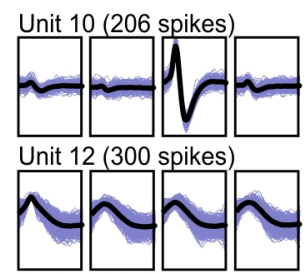

Unit 14 (825 spikes)

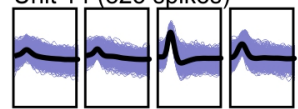

Unit 16 (788 spikes)

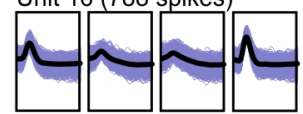

Figure 4. Validation of the automated framework with hippocampal recordings. a, Schematic depicting tetrode placement in the CA1 region (indicated by yellow oval) and recording paradigm in the linear track. b, Representative waveform overlays of single-units sorted using the automated framework. c, Waveform overlays of single-units sorted using the manual method. d, Top, number of single-units sorted per recording session using the automated framework (AF, green) or manual spike sorting (MSS, black). Bottom, amplitudes of mean waveforms sorted using the automated framework (green) or manual method (black). 

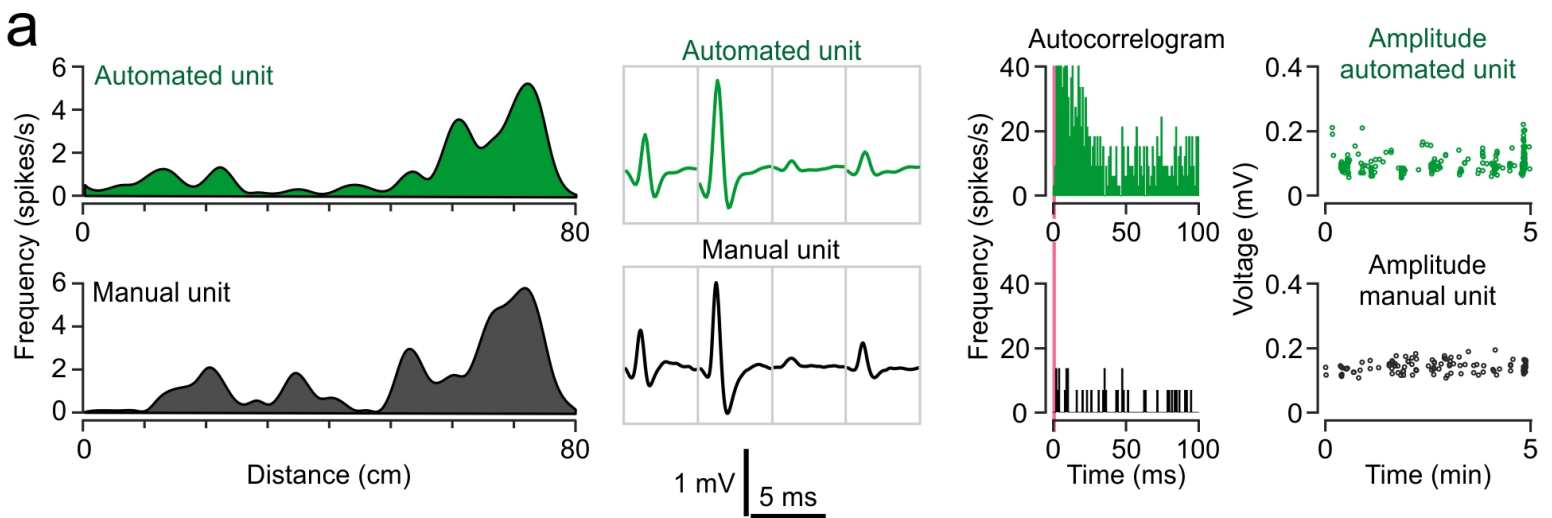

b
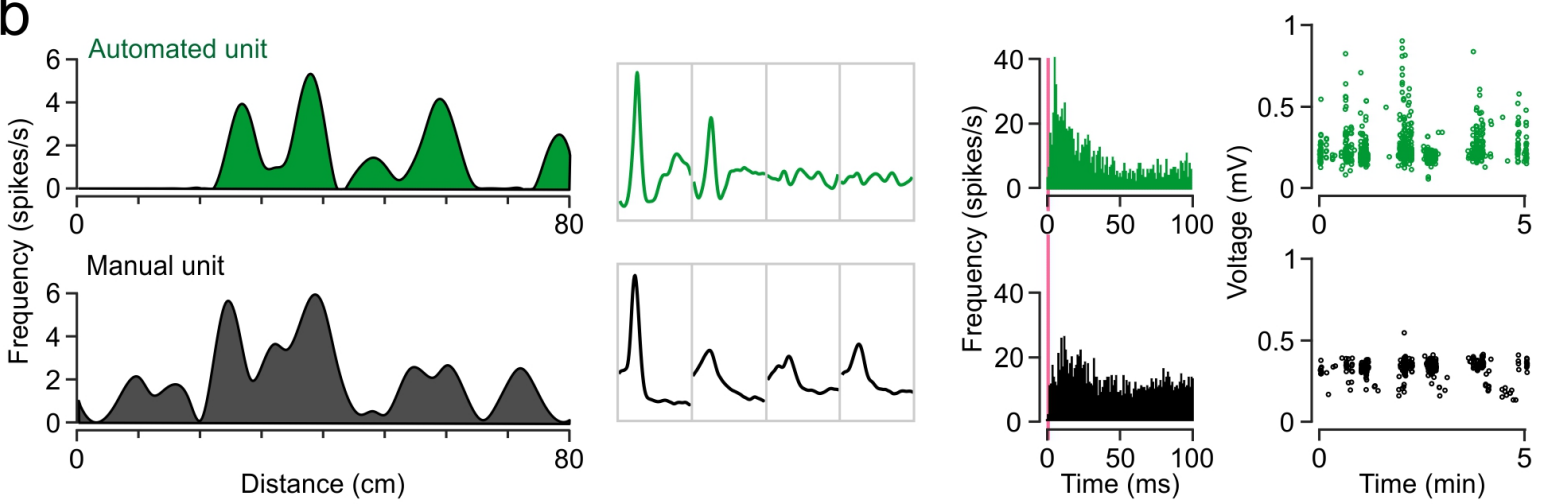

C

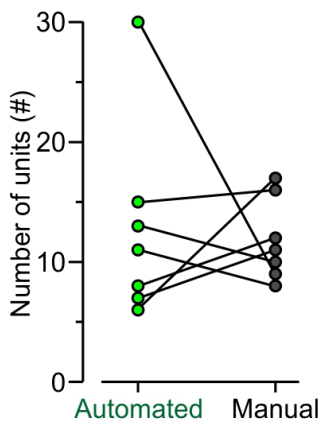

d

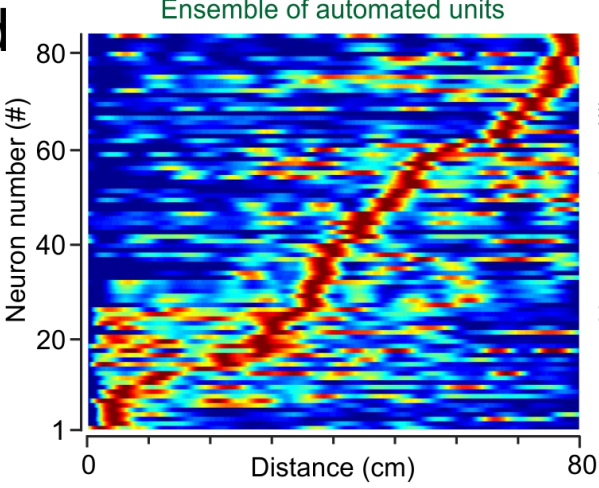

ensemble of manual units

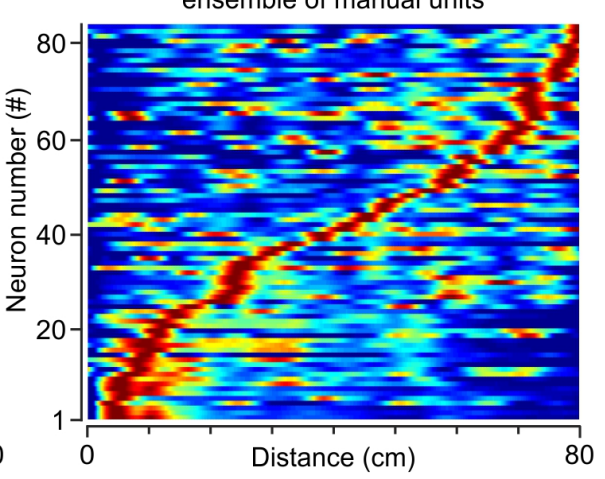

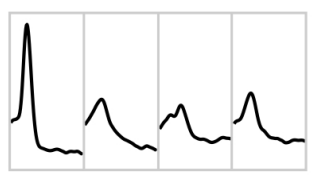

Figure 5. Hippocampal CA1 recordings in freely-moving mice. a, b, Representative single-units showing the firing rates along the length of the track (left panels), average waveform in each channel of a tetrode (next-to-left panels), autocorrelogram (next-to-right panels; pink lines represent refractory period), and amplitudes of spikes over time (right panels). Automated units are displayed in green and manual units are shown in black. a, Representative single-units likely representing the same putative neuron sorted with automated and manual methods. b, Similar units likely to represent different neurons across automated and manual methods. c, Number of single-units per recording session using the automated framework (green) or the manual method (black). d, Population of place cells recorded from mice $(n=4)$ running the linear track. Each row represents normalized spike activity of one single-unit, where rows are organized by the position of the peak firing rate for each unit along the linear track. Sorted populations of place cells appear similar across automated and manual methods. 


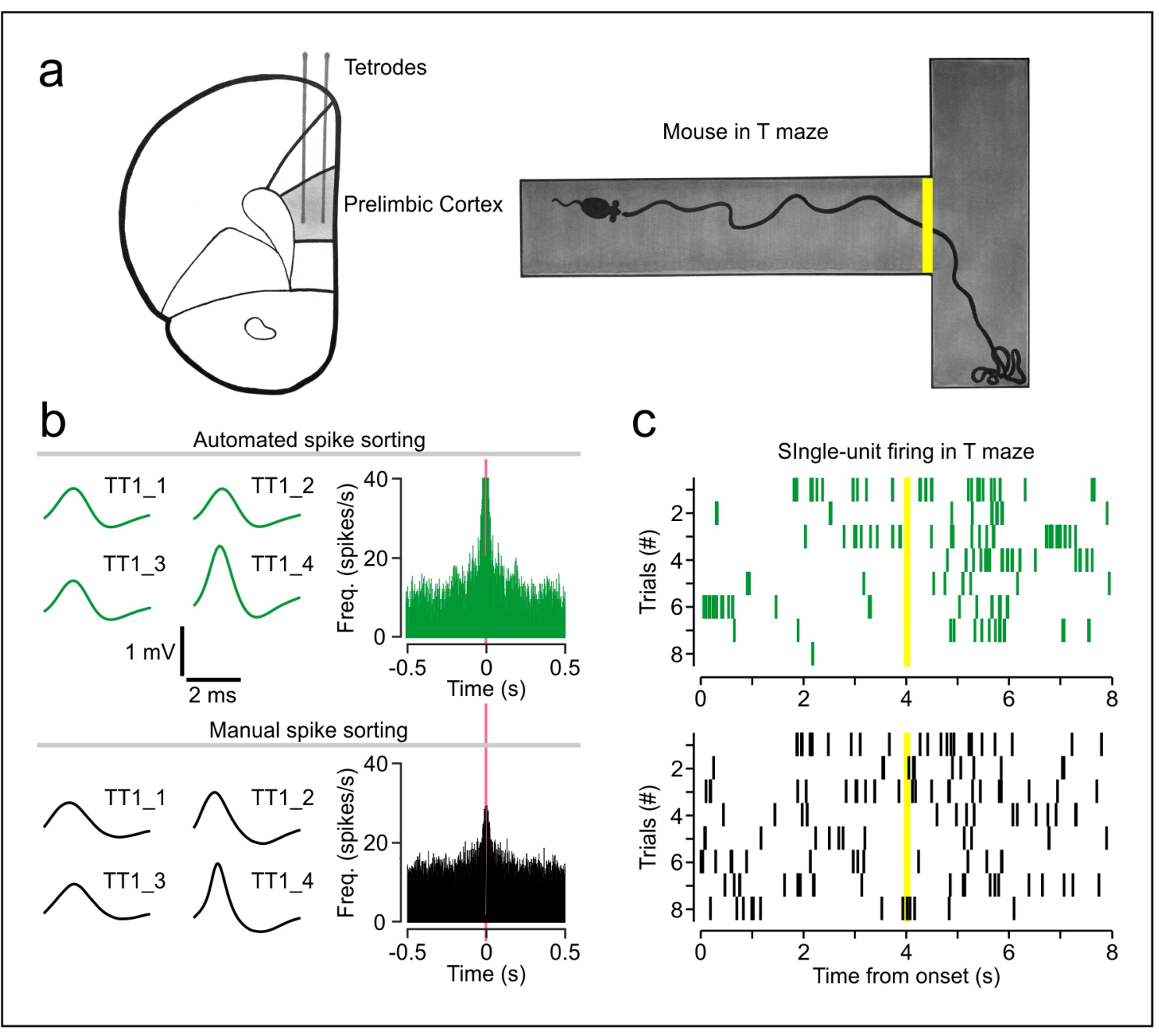

Figure 6. Prelimbic cortex recordings in freely-moving mice. a, Schematic depicting tetrode placement in the PL cortex and recording paradigm in the T maze. The yellow line indicates the point at which the mouse chooses to go left or right. b, Representative average waveforms and autocorrelograms of sorted units, likely representing the same putative neuron, across automated and manual methods; TT1_1, and so on, indicate tetrode channel numbers. The pink line represents the refractory period. c, Raster plots for singleunits in panel b. Each row represents a trial in the T maze and each tick is a spike. Notice that the trials are aligned to the timepoint indicated by the yellow line $(4 \mathrm{~s})$, which corresponds to the moment that the mouse arrives at the intersection of the stem with the arms of the T maze in each trial (as in panel a). 